\title{
Letter to the Editor: Increased Circulating Angiopoietin-like Protein 8 Levels Are Associated with Thoracic Aortic Dissection and Higher Inflammatory Conditions
}

\author{
Xiaoming Jia ${ }^{1}$ \\ Published online: 4 May 2020 \\ (C) Springer Science+Business Media, LLC, part of Springer Nature 2020
}

To the Editor:

I read with great interest the article by Yang et al. on the association between angiopoietin-like protein 8 (ANGPTL8) with thoracic aortic dissection (TAD) [1]. The authors should be commended for a comprehensive study that includes assessment of serum ANGPTL8 levels as well as tissue specific expression and in vitro experiments. However, there are a few aspects that require clarification.

The authors concluded that ANGPTL8 may be a predictor of TAD. It is unclear what the authors intended with this point. Do they mean that this biomarker would be a predictor of short- or long-term risk for TAD, that it may be used as a predictor of post-dissection complications or if it may be used to rule in or rule out dissections in a diagnostic scenario? While the authors provide AUC calculations comparing ANGPTL8 with d-dimer and hs-CRP, it is unclear how these calculations can be applied practically in a clinical setting. Moreover, if the intent is to assess long-term risk prediction of TAD using ANGPLT8, the current study design may be limited in addressing this question.

Secondly, I find it surprising that the authors do not adjust for smoking in their regression models. Smoking has known association with inflammation and is a risk factor for aortic aneurysm/dissection $[2,3]$. The authors should consider investigating whether smoking may impact the association between ANGPTL8 and TAD.

Xiaoming Jia

xiaoming.jia@bcm.edu

1 Baylor College of Medicine, Houston, TX 77030, USA

Lastly, it will be interesting if the authors can also assess the association between ANGPTL8 and thoracic aortic aneurysms without dissection. As the authors have shown, there is association of serum biomarker levels with TAD and increased expression of ANGPTL8 in TAD tissue samples. Extension of this association to aortic aneurysms may provide further insight into the relationship between ANGPTL8 and vascular health.

\section{References}

1. Yang Y, Jiao X, Li L, Hu C, Zhang X, Pan L, et al. Increased circulating angiopoietin-like protein 8 levels are associated with thoracic aortic dissection and higher inflammatory conditions. Cardiovasc Drugs Ther. 2020;34(1):65-77.

2. McEvoy JW, Nasir K, DeFilippis AP, Lima JA, Bluemke DA, Hundley WG, et al. Relationship of cigarette smoking with inflammation and subclinical vascular disease: the multi-ethnic study of atherosclerosis. Arterioscler Thromb Vasc Biol. 2015;35(4):100210.

3. Goldfinger JZ, Halperin JL, Marin ML, Stewart AS, Eagle KA, Fuster V. Thoracic aortic aneurysm and dissection. J Am Coll Cardiol. 2014;64(16):1725-39.

Publisher's Note Springer Nature remains neutral with regard to jurisdictional claims in published maps and institutional affiliations. 